\title{
Aus der Praxis.
}

\author{
Von \\ J. Holst in Dorpat.
}

\section{Empfängniss während der Behandlung mit einem Intranterinpessarium.}

Im Jahre 1871 berichtete O1shausen über zwei Beobachtungen, in denen, während intrauterine Stifte getragen wurden, Schwangersèhaft eingetreten war. In beiden Fällen nahm die Schwangerschaft einen regelmässigen Verlauf. Diesen beiden Beobachtungen konnte Winckel im Jahre 1872 einen dritten Fall hinzufügen, in welchem aber nach Entfernung des Stiftes im dritten Monate der Schwangerschaft Abortus eingetreten war.

Im Jahre 1873 hatte ich Gelegenheit, eine ganz analoge Beobachtung zu machen, die bei der Seltenheit derselben hier mitgetheilt werden soll.

Im November 1872 iibernahm ich die Behandlung der Frau $\mathbf{F}$., welche, seit wenigen Monaten verheirathet, das Bild vorgerückter Anämie und ausgeprägter Nervosität darbot. Patientin klagte über Schmerzen im Kreuze und Unterleibe, Schmerzen, welche zur Zeit der Menstruation einen besonders hohen Grad erreichten; nach der Verheirathung hatte sich reichlicher Fluor albus und Pruritus hinzugesellt, die Verdauung war träg, die Harnentleerung oft schmerzhaft.

Die Untersuchung des Genitalsystems der im Uebrigen gesunden. Frau ergab Katarrh der Vaginal- und Cerviealschleimhant; den Uterus von normaler Grösse, recktwinkelig nach vorn geknickt und frei beweglich; das Beckenbindegewebe war nicht erkrankt, die Eierstöcke von normaler Beschaffenheit.

Nachdem der Katarxh der Vaginal - und Cervicalschleimhaut beseitigt waren, ging ich zur instrumentellen Behandlung der Flexion des Uterus iiber. In die Gebärmutter wurde ein Hartkautschukstift, getragen von einem runden Knopfe von einem Zoll Durchmesser und einem halben Zoll Dicke, eingeführt. Dieses Intrauterinpessarium fixirte ich auch in diesem Falle, wie auch sonst immer durch ein Hartkautschuk-Hebelpessarium. Es ist das die mildeste und zugleich eine ganz sichere Methode der Fixirung. Indem das Hebelpessarium, ganz so angewandt wie bei Retroversionen, die Vaginalportion nach hinten zieht, drängt es dieselbe gegen die hintere Scheidenwand, welche wiederum den den Stift tragenden Knopf gegen den äusseren Muttermund andrückt und das Herabgleiten des Intrauterinpessariums 
verhindert. Die mildeste Methode ist diese, weil sie die freieste Beweglichkeit des Organes gestattet.

Das Intrauterinpessarium lag vortrefflich, verursachte nicht die leisesten Beschwerden, und der Erfolg war so gut man denselben nur wünschen konnte. Zunächst schwanden die Schmerzen, die Stuhlverstopfung, die Harnbeschwerden; die Menses verliefen ohne Schmerzen; Schlaf und Appetit kehrten wieder, die hysterischen Erscheinungen und die Anämie besserten sich auffallend schnell. Anfang März fühlte sich die Kranke so wohl, wie sie in Jahren nicht gewesen war, hielt sich für vollkommen genesen, und wünschte dringend, aach Hause entlassen zu werden. Ich konnte diesem Wunsche um so leichter nachgeben, als Frau F. in der Nähe ihres ländlichen Wohnortes nöthigen Falls jeden Tag tïchtige ärztliche Berathung haben konnte. - Das Intranterinpessarium sollte nach meiner Verordnung wenigstens ein Jahr getragen werden.

Anfang Juli 1873 sab ich auf einer Ferienreise Frau F, wieder. Sie berichtete, dass am 14. April die letzte Menstruation eingetreten sei, und in einem Briefe theilte mir Herr Dr. Hildebrandt mit, dass er, weil er Schwangerschaft vermuthete, am 12. Juni den Stift und das Hebelpessarium entfernt habe. „Das Intrauterinpessarium „nahm unverxückt und fest die ihm zukommende Lage ein; der "Knopf sass dicht am äusseren Muttermunde, und ich konnte den "Apparat nur mit einiger Schwierigkeit entfernen," meldete mir" Dr. Hildebrandt, und wünschte, dass ich durch eine Untersuchung die Existenz der vermutheten Schwangerschaft bestätigen sollte.

Ich konnte keinen Augenblick an der Richtigkeit seiner Diagnose zweifeln. Es lag Schwangerschaft in der zweiten Hälfte des dritten Monates vor. Die Schwangerschaft nahm ihren regelmässigen Verlauf; am 4. September wurde die erste Kindesbewegung gefühlt; am 23. Januar 1874 wurde nach achtstündiger Geburtsarbeit ein sehr kräftiger lebender Knabe geboren. Das Wochenbett verlief normal.

Recapitulire ich die einzelnen Daten, so wurde acht Wochen nach der letzten Menstruation der Stift entfernt; elf Wochen nach der letzten Menstruation und drei Wochen nach Entfernung des Stiftes konnte ich zweifellos die zweite Hälfte des dritten Schwangerschaftsmonates constatiren, und meine Diagnose Frau F. und Dr. Hildebrandt mittheilen; 282 Tage nach dem Beginne der letzten Menstruation wurde das Kind geboren.

Ueber die Art und Weise, wie in diesem Falle der Same in den Uterus gelangte, und bei den früheren bis jetzt publicirten Beobachtungen, möchte hier zu speculiren noch nicht an der Zeit sein, so lange über diesen Hergang unter normalen Verhältnissen die Ansichten noch so weit auseinander gehen. 


\section{Geschichte eines Myoma uteri.}

"Geschichte eines Myoma uteri" glaubte ich die vorliegende Beobachtung überschreiben zu müssen, weil dieselbe. für mich und die Fachgenossen nicht sowohl. durch die Grösse des Myoms und durch den Erfolg der eingeleiteten Behandlung von besonderem Interesse ist, als vielmehr durch die schliesslich eintretende vollständige Rückbildung eines Myoms, welches den Uterus bis zu dem Umfange des sechsten Monates der Schwangerschaft ausgedehnt hatte.

Frau X. wandte sich an mich vor jetzt 16 Jahren. Dieselbe hatte 12 Jahre vorher im 23. Lebensjahre nach normalem Verlaufe ihrer ersten Schwangerschaft in leichter Geburtsarbeit einen gesunden Knaben geboren, den sie selbst säugte. Wenige Jahre darauf stellten sich bei der bis dahin ganz gesunden. Frau Erscheinungen von Gebärmutterleiden ein. Die Menstruation wurde schmerzhaft, die freien Intervalle wurden kürzer; die Frau blieb unfruchtbar. Ich fand im Beginn der Behandlung bei ein und der anderen Menstruation noch Fetzen von der Decidua menstrualis. Die lokale Untersuchung ergab den Uterus anteflectirt, mässig vergrössert, Katarrh. Achtzehn Monate nach dem Beginne der Behandlung trat Schwangerschaft ein; dieselbe wurde in der ersten Hälfte durch heftige Schmerzen gestört, welche sich zu der Zeit einstellten, wo die Menstruation hätte eintreten sollen, und mehrmals Abortus befürchten liessen. $\mathrm{Zu}$ Blutungen kam es nicht. Die Geburt trat rechtzeitig ein und verlief normal; am 4. Tage Schüttelfrost und peritonitische Schmerzen, nach wenigen Tagen vollständige Genesung. Das kräftige Mädchen wurde nach wenigen Wochen einer Amme ïbergeben. Bald nach dem Entwöhnen stellte sich die Menstruation ein, diese und die späteren verliefen normal, und über die früheren Uterinbeschwerden wurde nicht mehr geklagt: Etwa ein Jahr nach der letzten Entbindung erfolgte im zweiten Monate der Schwangerschaft Abortus, nach welchem sich alle früheren Uterinbeschwerden wieder einstellten; Blutungen, Unregelmässigkeit, Schmerzen, nur die Membranen fehlten. Die Untersuchung ergab den Uterus vergrössert, derb, auch der Katarrh war wieder da, eine Knickung konnte jetzt nicht nachgewiesen werden. Alle therapeutischen Versuche, eine Verkleinerung des erkrankten Organes herbeizuführen und die bedeutendsten Symptome wenigstens zu mildern, blieben erfolglos. Das Leiden wurde von mir als chronischer Infarct aufgefasst, ebenso von einem der ersten deutschen Gynäkologen, der consultirt wurde. Badecuren in Kissingen und Brückenau, Aufenthalt in Seelisberg, St. Moritz und am Seestrande besserten vorübergehend das Allgemeinleiden. Die lokalen Erscheinungen, Schmerzen und Blutungen wurden schlimmer. - Der Umfang des Uterus nahm langsam aber stetig zu. Als derselbe etwa den Umfang des Uterus in der zehnten Schwangerschaftswoche erreicht hatte, wurde in dem Untersuchungsbefunde die Veränderung bemerkbar, dass sich namentlich die hintere Wand des Uterus über dem inneren Muttermunde stark vorwölbte, und konnte es nicht mehr 
zweifelhaft sein, dass es sich um eine Neubildung in der Höhle des Uterus handelte, besonders da auch die Sonde eine bedeutende Verlängerung der Höhle nachwies. Die sehr profuse Menstruation, die anhaltenden, sehr heftigen Schmerzen, der bei diesem Unfange des Uterus noch gänzlich mangelnde Einfluss auf Eröffnung des inneren Muttermundes und Veränderung des Scheidentheiles liessen mich nicht daran mweifeln, dass es sich um ein nicht gestieltes, breit anfsitzendes submucöses, vielleicht noch interstitielles Myom handelte, bei dem an eine Operation nicht zu denken war. Die Sondenuntersuchung ergab den Sitz in der vorderen Wand des Uterus. Die Aufschliessung des Kanales und des inneren Muttermundes bestätigten die Diagnose. - Der Uterus nahm rasch und bedeutend an Umfang zu. Die Blutungen wurden stärker, hielten jetzt und auch in späterer Zeit immer einen recht regelmässigen Typus von 22-24 Tagen ein; die Schmerzen wurden immer quälender. Der Uterus hatte bis zum Mai 1867 etwa den Umfang des fünften Schwangersschaftsmonates erreicht. - Es wurde nun, nachdem in Deutschland, namentlich durch Spiegelberg, die Spaltnng der Schleimhaut des Cervix und des unteren Uterusabschnittes empfohlen worden war, diese Operation dureh Simon aus Rostock ausgeführt, und bald darauf mit Injectionen von Liq. ferri sesquichlorati begonnen. Vermittels der Braun'schen Spritze wurde der Liquor, mit gleichen Theilen Wassers verdünnt, einen Tag um don anderen in den Uterus injicirt. $\mathrm{Da}$ ich längere Zeit von Dorpat abwesend sein musste, übernahm Dr. Bidder die Behandlung, die nach Uebersiedelung desselben nach Petersburg wieder in meine Hầnde überging. Die Finspritzongen wurden einen $\mathrm{Tag}$ um den anderen vorgenommen, zur Zeit der Blutung täglich, und blieben weg nur am ersten Tage der Blutung, die noch immer einen fast ganz regelmässigen Typus einhielt, der dem Menstruationstypus des Krankheitsbeginnes entsprach. Die Einspritzungen erregten für die ersten Stunden nicht unbedeutenden Schmerz, nicht eine einzige aber hat während der ganzen Dauer der Behandlung eine bedenkliche Reaction hervorgerufen. In der oben geschilderten Weise wurde die Behandlung bis in den Herbst fortgeführt. Die Blutungen wurden jetzt mässiger; die Injectionen konnten seltener gemacht werden, und wurden in den folgenden zwei Jahren nur noch vorgenommen, wenn eine oder die andere Blutung besonders heftig auftrat, und hier stets mit dem besten Erfolge. Im Jahre 1869 blieben im 47. Lebensjahre der Kranken diese menstruellen Blutungen ganz aus, nachdem dieselben schon ein Jahr unregelmässig meist in längeren Pausen sich eingestellt hatten. Der Uterus erreichte jetzt den Umfang eines Uterus am Ende des sechsten Schwangerschaftsmonates. Die Portio vaginalis verhielt sich wie im Beginn der Erkrankung. Seit dem Aufhören der Menses sind jetzt bald fünf Jahre verflossen. - In den ersten Jahren nach dem Aussetzen der Menses war keine deutliche Volnmenabnahme des Uterus nachzuweisen, später aber wurde dieselbe, wenn nach längeren Intervallen untersucht wurde, sehr bemerkbar. - Jetzt, also nach fünf Jahren, hat der Uterus die Grösse 
eines Uterus in der 6. Schwangerschaftswoche, und sind alle Beschwerden von Seiten des Genitalsystems geschwunden. Es ist vielleicht noch der Erwähnung werth, dass in den letzten fünf Jahren fast ununterbrochene Neigung zu Durchfällen bestand, die nur durch anhaltenden Gebrauch von Clystiren von Argent. nitr. zeitweilig beseitigt werden konnten.

Ob die Neubildung schon vor 16 Jahren, als ich die Behandlung uibernahm, vorhanden war und die Ursache der Knickung abgab; ob die Besserung des Uterusleidens und das Schwiaden der Knickung nach Schwangerschaft und Wochenbett auf theilweise erfolgte Resorption im Wochenbette zu beziehen sind, ob der dann erfolgte Abortus, das Zunehmen der alten Beschwerden auf ein neues Wachsthum des Myoms zu beziehen ist; das sind Fragen, die sich jetzt nicht beantworten lassen, wenn auch die Wahrscheinlichkeit eines solchen $\mathrm{Zu}$ sammenhanges nicht in Abrede gestellt werden kann. Zweifellos ist aber, dass das Myom fast 10 Jahre brauchte, bis es den bedeutenden Umfang erreichte, der eben angegeben wurde. Von dem grössten Interesse ist aber jedenfalis, die wenn auch nicht einzig dastehende gewiss aber selten gemachte Beobachtung einer vollständigen Resorption eines Myoms von dem Umfange, in der Zeit der Decrepidität, wenn anch fünf Jahre dazu nöthig waren. Ich sage ,, vollständig"; denn soweit ist derselbe wenigstens für die Kranke geschwunden, die keine Andeutung von Uterusbeschwerden mehr hat; ob aber für den pathologischen Anatomen die Involution eine vollständige ist, kann so lange in Zweifel gezogen werdeu, als der Uterus noch einen die normalen Maasse überschreitenden Umfang bietet, und dürfte mit Sicherheit erst dann behauptet werden, wenn er die charakteristischen Veränderungen des decrepiden Uterus bieten wird. Wenn aber noch Residuen des Myoms vorhanden sind, so sind es jedenfalls nur noch Bindegewebsreste von ganz unbedentendem Umfange. - Wie weit die energische und lange fortgesetzte Behandlung mit den Injectionen von Erfolg gewesen, lässt sich wohl mit Sicherheit feststellen. Der Einfluss auf Verringerung und Verkürzung der jedesmaligen Blutung ist wohl nicht in Abrede zu stellen; ebensowenig wird sich daran zweifeln lassen, dass die Injectionen durch theilweise Verödung und Schrumpfung der Schleimbaut und ihrer Gefässe den Erfolg hatten, dass schon vor dem Aufhören der Menstruation die Blutverluste so mässig wurden, dass es nur selten einer Injection bedurfte. Auch das bei einem derartigen Leiden frühe Aufhören der menstruellen Blutungen im 47. Lebensjahre möchte ich für Wirkung der Behandlung halten. Möglich ist, dass dieselben auch das Wachsthum beschränkt haben.

Schliesslich darf ich nicht unterlassen hinzuzufügen, dass weder Dr. Bidder noch ich bei dieser Behandlung es jemals für nöthig gefunden haben, vor den Injectionen den Cervicalkanal und das Os internum zu erweitern, und dass die mässige Reaction trotz der Unterlassung dieser von vielen für nöthig erachteten Vorsichtsmassregel sich nicht dadurch erklärt, dass vor dem Beginne der In- 
jectionen eine Spaltung vorgenommen war; denn der Kanal und das Os internum waren bei der Behandlung stets so eng wie vorher, und war das Einfïhren der Spritze immer recht schwer und nur bei einer ganz besonderen Krümmung des Injectionsrohres möglich. Ich füge noch hinzu, dass ich nie vor den Injectionen erweitere, ich habe vielmehr von dem Gebrauche der Laminaria und des Pressschwammes zum Zwecke der Erweiterung wohl hier und da eine Reaction von der Heftigkeit gesehen, wie sie mir nach Injectionen selbst concentrirter Flüssigkeiten in die Höhle des Uterus nie vorgekommen ist, wenn nur die Vorsicht gebraucht wurde, dass mit reinem Wasser oder ganz schwachen Lösungen begonnen, und je nach der Empfindlichkeit schneller oder langsamer zu concentrirteren Lösungen äber'gegangen wurde. Es kommen Fälle vor, wo schon die Injectionen von reinem Wasser so empfindlich sind, dass man von der Behandlung mit Injectionen von Flüssigkeiten abstehen muss; aber in diesen Fällen muss man auch von der Erweiterung mit Laminaria und Pressschwamm abstehen, und hat man diese auch durchgesetzt, die Empfindlichkeit bleibt nach der Erweiterung gegen die Injectionen dieselbe.

\title{
Ein weiterer Fall spontanen Schwundes eines Uterusmyoms.
}

\author{
Von \\ Otto Spiegelberg.
}

Die im Vorhergehenden mitgetheilte Beobachtung des Hrn. Holst. giebt mir Veranlassung, über eine höchst ähnliche von spontanem rölligen Verschwinden eines intraperietalen Myoms zu referiren. Sie ist in Kürze folgende.

Frl. B., 46 J. alt, Virgo, consultirte mich im Nov. 1871, weil ihre Menstruation seit $2^{1 / 2} \mathrm{~J}$. immer reichlicher, in den letaten $\mathbf{M}_{0-}$ naten profus und von 14täg. Dauer geworden, und weil sie im Unterbanche eine Geschwulst wahrgenommen. Die anämische, sonst aber kräftig gebaute Dame, hatte einen Uterus vom Unfange des 4 Mon. schwangeren; die Vergrösserung war eine ziemlich gleichmässige des Corpus, nur die linke Seite desselben war nach aussen vorgebaucht; die Consistenz sehr elastisch; Cervix lang und ganz geschlossen. Beweglichkeit frei, keine Deviation. - Während der nächsten sehr 